\title{
Development of a Dynamic Symbiotic Network Planner and Application to a Living Lab Testbed
}

\author{
David Plets*, Wout Joseph, Kris Vanhecke, Luc Martens \\ WiCa-IBBT, Dept. of Information Technology, UGent \\ Email: david.plets@intec.ugent.be \\ Eli De Poorter, Bart Jooris, Ingrid Moerman \\ IBCN-IBBT, Dept. of Information Technology, UGent \\ G. Crommenlaan 8 bus 201 \\ 9050 Ghent, Belgium
}

\begin{abstract}
The concept of symbiotic networks is introduced as a way to improve the use of available resources and infrastructure and the overall performance of co-located wireless networks. An existing wireless network planning tool is extended with a symbiotic feature, which is able to create a new (symbiotic) network out of the different coexisting networks. This symbiotic planning tool is applied to a living lab testbed network in Ghent, Belgium. Feedback about reception quality at the testbed nodes is continuously given to the tool and the internal propagation prediction models are adapted accordingly in order to improve the accuracy of the network planning. The concept of this dynamic symbiotic network planner are presented.
\end{abstract}

\section{INTRODUCTION AND OBJECTIVES}

In recent years, indoor wireless networks have become omnipresent wherever the need for communication arose. This has led to the existence of many different co-located wireless technologies: GSM (Global System for Mobile Communications), UMTS (Universal Mobile Telecommunications System), Bluetooth, Zigbee, DECT (Digital Enhanced Cordless Telecommunications), WiFi, LTE (Long Term Evolution),... The increase in the density of wireless devices, each using their own technology and having their own limitations (battery lifetime, memory capacity,...), leads to an increasing amount of interference and a sub-optimal use of available resources. Symbiotic networks [1], coexisting homogeneous (originally using the same technology, e.g., two independent WiFi networks) and heterogeneous (e.g., WiFi and UMTS networks) networks that cooperate based on common incentives through infrastructure and resource sharing, can provide benefits for all participating networks. Sharing wireless network infrastructure between different wireless networks could increase QoS (Quality of Service), spectrum use efficiency, energy efficiency, ... In this paper, the concept of symbiotic networks is implemented in an indoor path loss prediction tool, in order to perform the network planning of the desired symbiotic network. As a more advanced feature, information about the reception quality at the level of the network receiver devices is fed back to the tool to create a dynamic symbiotic network planner, where prediction errors could be resolved by adapting the internal path loss models.
In this paper, a dynamic symbiotic network planner will be presented, where the symbiotic networking concept is implemented in the WHIPP (WiCa Heuristic Indoor Propagation Prediction) tool, a path loss prediction tool based on the dominant path model, created in cooperation with usability experts [2]. The tool is implemented as a web service with a Java engine that allows the user to draw or import the ground plan of a building and predict the coverage for a predefined receiver device in the different rooms on a floor level for a given configuration of transmit devices. The concept and of the symbiotic application of this planning tool to a living lab testbed network in Ghent, Belgium will be presented.

The outline of this paper is as follows. In Section II, the living lab testbed network will be presented, and Section III will go more deeply into the concept of symbiotic networks. Section IV briefly discusses the network planning tool. In Section V, the symbiotic network planner will be applied to the living lab testbed network. Finally, Section VI presents the conclusions of this paper.

\section{LIVING LAB TESTBED NETWORK}

200 nodes, equipped with 2 Wi-Fi IEEE 802.11 interfaces $(\mathrm{a} / \mathrm{b} / \mathrm{g})$ and 1 or 2 CC2420 sensor nodes [3] with IEEE 802.15.4 interface embedded with temperature, light, and humidity sensors, have been put up at a height of $2.5 \mathrm{~m}$ over 3 floors of an office building in Ghent, Belgium. The sensor chip is an RF (Radio Frequency) transceiver designed for low-power and low-voltage wireless applications and has a programmable output power, varying in 8 steps between $25 \mathrm{dBm}$ and $0 \mathrm{dBm}$ [3]. In receiving mode, the Received Signal Strength Indication (RSSI) indicates the received power and is a good indicator for the packet reception rate (PRR) when the noise is limited [4]. Fig. 1 shows the location of all the nodes of this living lab testbed network on the third floor $(90 \mathrm{~m} \times 17 \mathrm{~m})$ of the investigated office building. The nodes belong to three different sensor networks, indicated with a different color and a different letter inside the marker (A, B, or $\mathrm{C})$. 


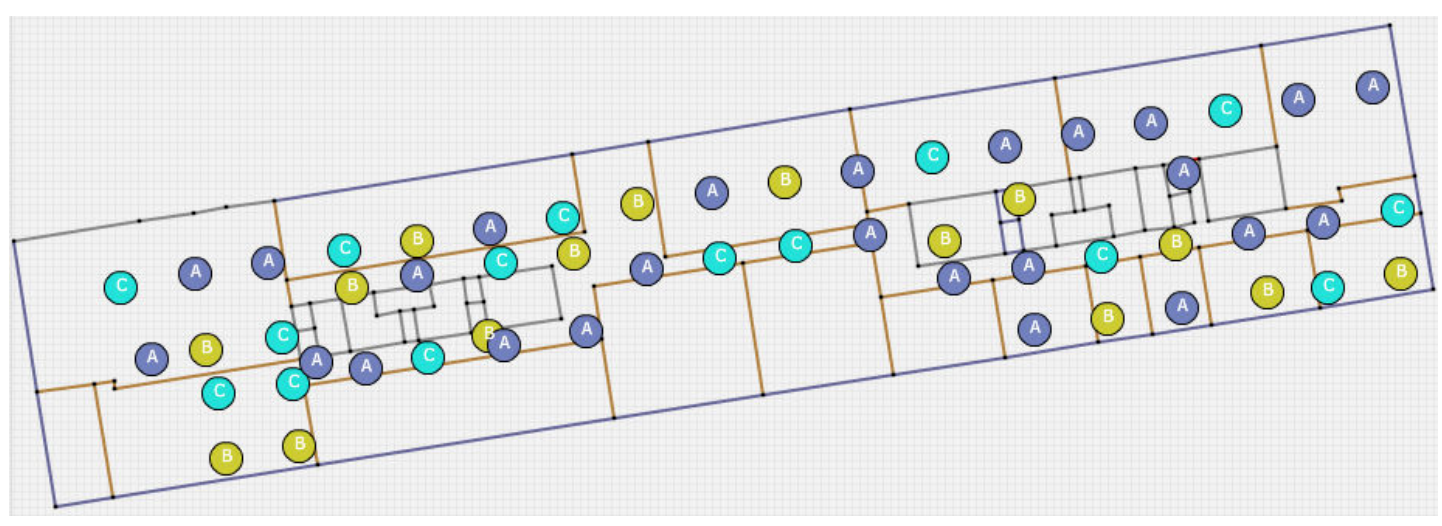

Fig. 1. Third floor of office building with indication of nodes belonging to the three different networks (A, B, and C)

\section{SYMBIOTIC NETWORKS}

Symbiotic networks is a promising concept in a world where a lot of wireless technologies coexist independently from each other. Two WiFi networks from two different companies can e.g., share access points, allowing to decrease interference and improve coverage with a lower energy consumption (cooperation of homogeneous networks). Another example is a smartphone user collecting data over WiFi instead of over UMTS, whenever the user is located in a suitable WiFi network (cooperation of heterogeneous networks). It is clear that the creation of symbiotic networks allows to use available resources much more efficiently. Independent networks can decide to cooperate based on common incentives, which can vary in the course of time. Possible incentives are a lower energy consumption, lower exposure, a longer device life time, lower interference, a higher throughput, a better QoS (Quality of Service) ... However, while striving to meet each one of these incentives, it is crucial that the network remains operative, even when e.g., transmit powers are lowered for the purpose of energy consumption.

Therefore, we choose to implement the symbiotic networking concept into an indoor network planning tool, in order to perform the network 'replanning' of the desired symbiotic network. In a second phase, also signal quality parameters, recorded at the receiver devices, can be used to adjust and improve the symbiotic network planning, in this way creating a dynamic symbiotic network planner.

\section{WIRELESS NETWORK PLANNING TOOL}

The heuristic WHIPP tool has been developed and validated for the prediction of path loss in indoor environments [5]. It takes into account the effect of the environment on the wireless propagation channel and has been developed for the prediction of the path loss in zones of about $5 \mathrm{~m}^{2}$ or at specific locations. It bases its calculations on the dominant path between transmitter and receiver. This dominant path is determined with a multidimensional optimization algorithm that searches the lowest total path loss, consisting of a distance loss (taking into account the length of the propagation path), a cumulated wall loss (taking into account the walls penetrated along the propagation path), and an interaction loss (taking into account the propagation direction changes of the path, e.g., around corners). The tool has been applied to the $2.4 \mathrm{GHz}$ band. Fig. 2 shows an example of the path loss calculation for a floor with three $2.4 \mathrm{GHz} \mathrm{WiFi}$ access points (indicated with purple dots). The path loss or throughput in the rooms is displayed on the floor map with a colour code. The performance of the tool is validated with a large set of measurements in four buildings. In contrary to many existing tools no tuning of the tools parameters is performed for the validation. Excellent correspondence between measurements and predictions is obtained, even for other buildings and floors. As our tool is based on the free-space loss model for every environment, the tool is generally applicable, while other tools are often too dependent of the environment upon which the used propagation model is based. Our tool is offered to both professional and nonprofessional users through a web service, which allows easy access through an internet connection and an Adobe Flash player. The user interface is developed in cooperation with usability experts.

When the receiver devices are put up at specific, fixed locations (e.g., living lab node locations), path loss values become more unpredictable due to small-scale fading effects. However, feedback about the recorded path loss between a transmitting and a receiving node could cleverly be used to improve prediction models.

The path loss calculation feature described above is not only used to predict the coverage for a given set of transmit devices, it is also used as a basis for a node selection algorithm. This node selection algorithm selects a minimal number of transmit nodes out of a larger set, while still meeting a certain throughput requirement in specific rooms or at specific locations. Thus, existing networks are optimized by selecting nodes out of the total set, without affecting coverage. This algorithm is especially useful with a view on symbiotic networks, where the union of all co-located networks is reduced to one symbiotic network, consisting of nodes selected from the different networks. 


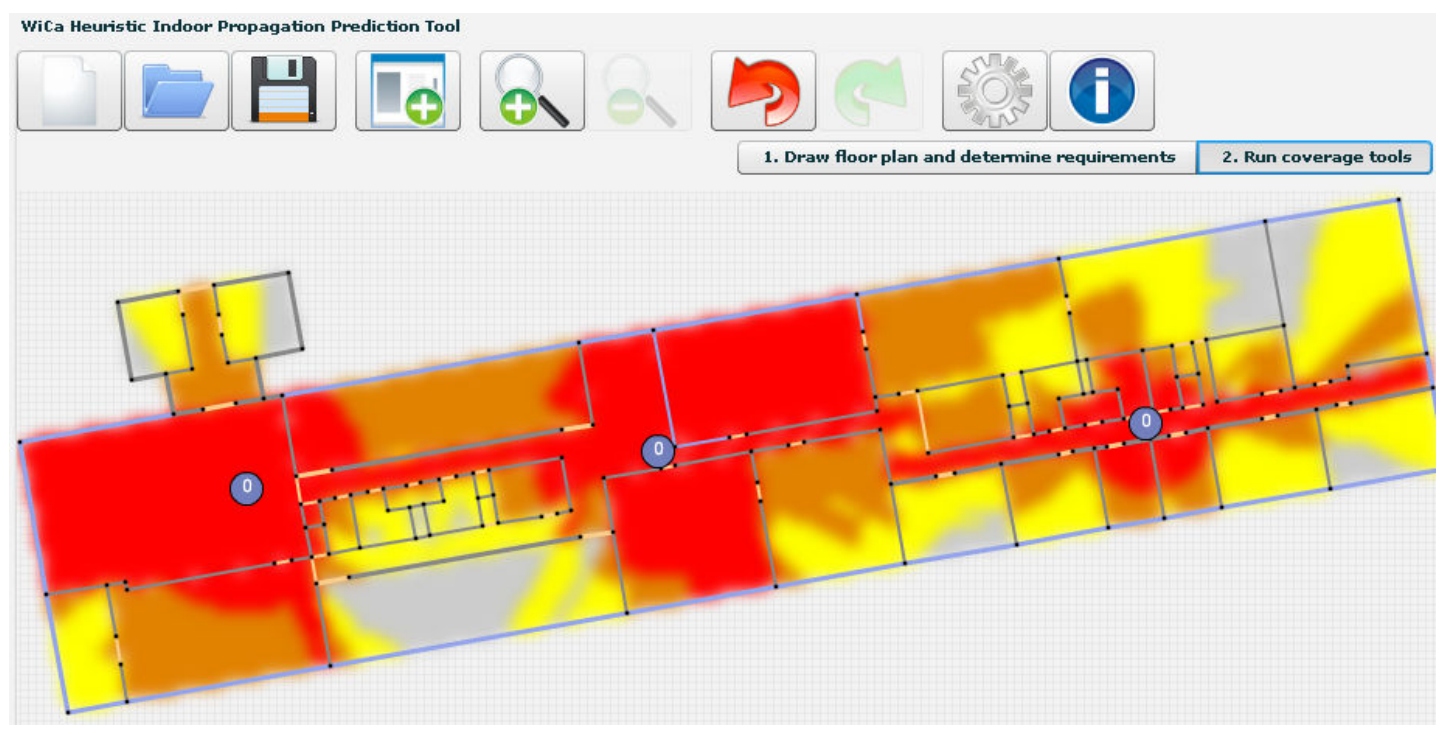

Fig. 2. Path loss calculation by the WHIPP tool

\section{APpliCATION TO LIVING LAB TESTBED NETWORK AND DYNAMIC MODEL ADJUSTMENT}

As a proof-of-concept, the symbiotic network planner is applied to the living lab testbed network described in Section II. The living lab network consists of three independent sensor networks A, B, and C (see Fig. 1). Each of the nodes of the different networks needs a sink node (i.e., a node that collects the data from the other nodes) to send its data to. To be able to always receive data from other nodes, a sink node should always be active. If the three networks would share their infrastructure, the number of sinks needed to gather all data could be limited and, as a consequence, also the energy consumption.

The planning tool uses its internal path loss models and the node characteristics to predict how many sinks are needed to be able to reach a sink from each of the nodes, and where these sinks should be located. Fig. 3 shows the resulting symbiotic network, containing only three sinks. The letters in the nodes now indicate to which sink the respective nodes send their data to (nodes with marker A send to sink 1, nodes with B send to sink 2, and nodes with $\mathrm{C}$ send to sink 3 ). The sink can either be a node from the sending node's own original network, or a node from another network.

Since the nodes are put up at fixed positions, the path loss between a sending node and a sink is subject to small-scale fading, making it more difficult to perform an accurate network planning. Therefore, we have included a feedback loop from the receiver devices towards the network planning tool, in order to adjust the tool's prediction models, based on the difference between the RSSI recorded at the receiver nodes and the RSSI predicted by the path loss model. After the adjustment of the model, the symbiotic network planning algorithm can be rerun, and a new set of sinks can be determined. Fig. 4 illustrates how this process can be repeated (until a certain predefined condition is met (e.g., average prediction error $<$ threshold)). The node selection algorithm of the WHIPP tool determines all node parameters (transmit power, on/off state,...) and while packets are sent accordingly, the signal quality parameters recorded at the receiving nodes are logged in a database. The planning tool then uses these parameters to adjust its prediction models and reapply the node selection algorithm, bringing us back at the start of the symbiotic network planning loop.

Another option is to not only adjust the prediction model while in the loop, but also adapt the node selection algorithm. This will be the case when the common incentives of the networks are redefined: e.g., when the networks require a lower exposure, the application of another node selection algorithm will be required. Finally, this dynamic network planning loop also allows to recover from a node failure.

A first test of the feedback loop has already been executed. After selecting the three sinks mentioned above, 100 packets were sent by each node to its corresponding sink and the measured path loss was compared with the predicted one. The first run yielded an average absolute prediction error $\mathrm{PL}_{\text {measured }}{ }^{-}$ $\mathrm{PL}_{\text {predicted }}$ of $7.3 \mathrm{~dB}$. Adapting the path loss model with a fixed offset of $2.9 \mathrm{~dB}$ (i.e., the value of the average prediction error) resulted in an average absolute error of $5.0 \mathrm{~dB}$ in the second run. This improvement of $2.3 \mathrm{~dB}$ indicates the usefulness of the feedback loop. In the future, new tests with more advanced adaptation strategies will be implemented. One has to keep in mind though, that the influence of small-scale fading will inevitably limit the prediction quality.

\section{CONCLUSION}

The concept of symbiotic networks is discussed: wireless networks with common incentives can share their resources and infrastructure and improve the overall performance. A network planning tool is presented and extended with a symbiotic feature, allowing to perform a network 'replanning' 


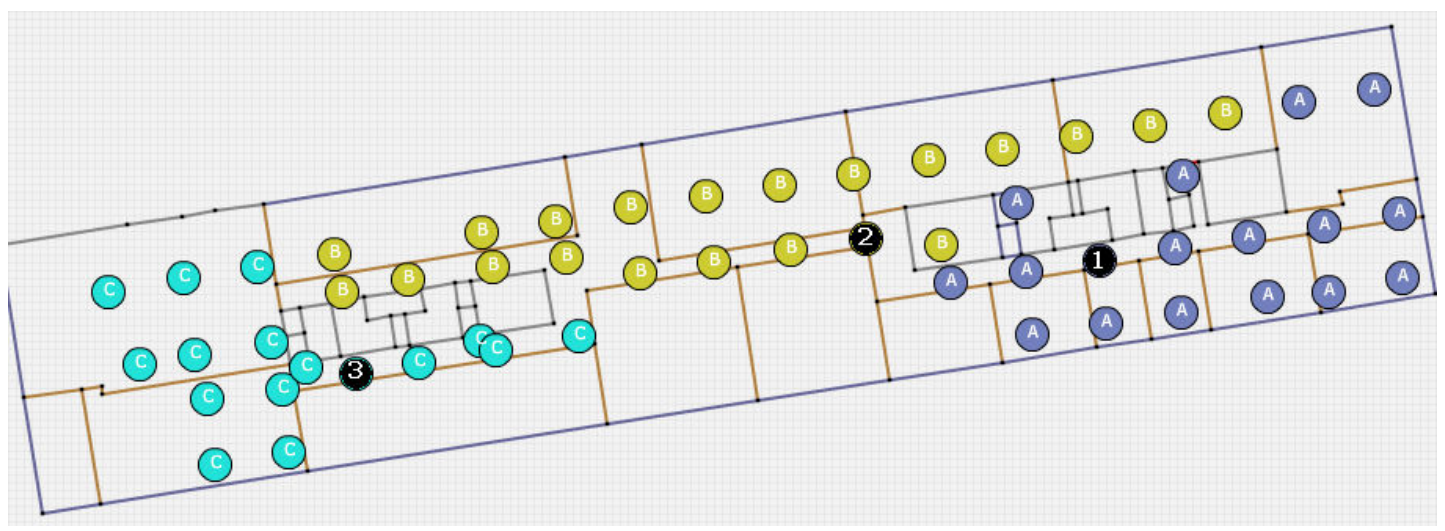

Fig. 3. Symbiotic network after node selection algorithm (sinks indicated with black dot and number, other nodes have indication of their sink: A- $>1$, $\mathrm{B}->2, \mathrm{C}->3$ )

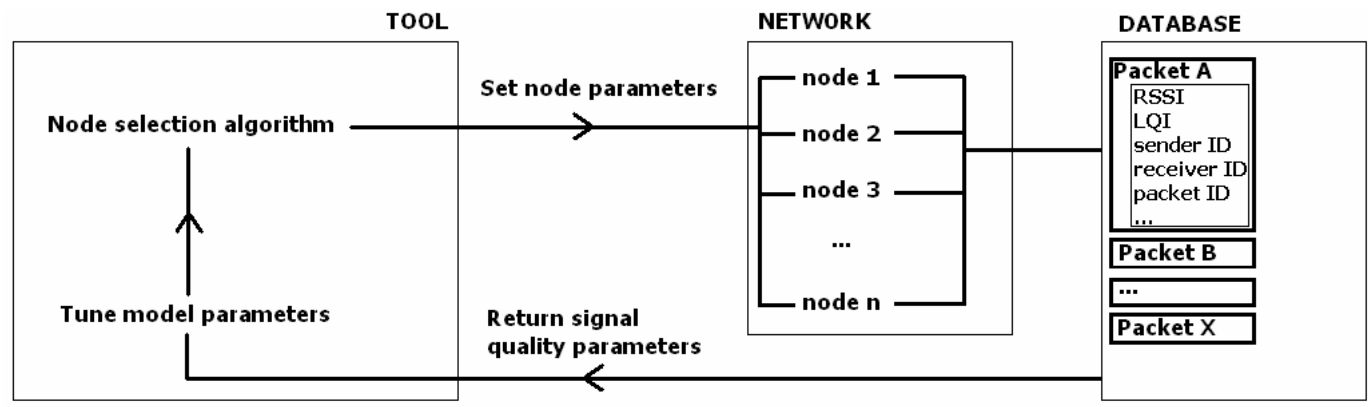

Fig. 4. Application of dynamic symbiotic network planner on living lab testbed network

of co-located wireless networks. This algorithm is applied to a living lab testbed network consisting of three sensor networks, in this way creating one symbiotic network with a lower total energy consumption. Signal quality parameters are logged at the receiver nodes and can be used to adjust and improve the predictions of the planning tool. A prediction improvement of $2.3 \mathrm{~dB}$ was already obtained in a first test.

\section{ACKNOWLEDGMENT}

This work was supported by the IWT-SBO SymbioNets project. W. Joseph is a Post-Doctoral Fellow of the FWO-V (Research Foundation-Flanders).

\section{REFERENCES}

[1] E. D. Poorter, B. Latre, I. Moerman, and P. Demeester, "Symbiotic networks: Towards a new level of cooperation between wireless networks," Special Issue of the Wireless Personal Communications Journal, vol. 45, no. 4, pp. 479-495, June 2008.

[2] D. Plets, W. Joseph, K. Vanhecke, E. Tanghe, and L. Martens, "Development of an Accurate Tool for Path Loss and Coverage Prediction in Indoor Environments," in European Conference on Antennas and Propagation 2010, Barcelona, 12-16 April 2010.

[3] Texas Instruments, "2.4 GHz IEEE 802.15.4 / ZigBee-ready RF Transceiver Datasheet," Tech. Rep. [Online]. Available: http://www.ti.com/lit/gps/cc2420

[4] K. Srinivasan and P. Levis, "RSSI Is Under-Appreciated," in Proceedings of the Third Workshop on Embedded Networked Sensors (EmNets), Cambridge, MA, May 2006.
[5] D. Plets, W. Joseph, K. Vanhecke, E. Tanghe, L. Martens, S. Bouckaert, I. Moerman, and P. Demeester, "Validation of Path Loss by Heuristic Prediction Tool with Path Loss and RSSI Measurements," in Antennas and Propagation Society International Symposium, 2010. AP-S 2010. IEEE, Toroto, Ontario, Canada, 11-17 July 2010. 